Methods: After professional training, rheumatologists use non-mydriatic fundus camera to take fundus photography of inpatients in our department and record the clinical features and laboratory test results of the patients. The results of fundus photography are interpreted by both ophthalmologists and rheumatologists. The fundus results of SLE patients from July 2016 to June 2017 were analysed. 35 cases (62 eyes) of retinopathy were defined as experimental group, and 35 cases (70 eyes) without retinopathy were randomly selected as the control group.

Results: A total of 203 patients with SLE with an average age of $38.4 \pm 11.6$ years acquired bilateral fundus results, including 28 males and 175 females (86.2\%). Ocular lesions were found in $51(25.1 \%)$ cases, of which $35(62.6 \%)$ were common retinopathy. There were 5 cases of retinal atrophy and pigmented degeneration (4 binocular, 1 monocular), 9 cotton spots (6 binocular, 3 monocular), 7 flaming bleeding (7 binocular), 14 choroiditis with drusen ( 9 binocular, 5 monocular), arteries tortuous or occluded in 5 (3 binocular, 2 monocular). The SLEDAI score, anti-dsDNA level and C3 decline rate of the experimental group were significantly higher than those of the control group $(p<0.05$ or $p<0.01)$, while the titer of antinuclear antibody, the positive rate of anti-SM antibody, the positive rate of anti-phospholipid antibodies, and the erythrocyte sedimentation rate had no significant difference $(p>0.05)$. Subsequently, we followed up 16 patients with retinopathy and SLEDAI score $>10$ and achieved remission (SLEDAI score $\leq 4$ ) with glucocorticoid and immunosuppressive agents with an average duration of 6.5 \pm 4.5 months. The second examination showed that retinal lesions were improved in 9 cases $(56.25 \%)$, 3 cases (18.75\%) were unable to judge due to the appearance of optical media change which may cased by glucocorticoid, and 4 cases (25\%) showed no obvious changes.

Conclusions: Non-mydriatic fundus examination performed by rheumatologists may assist rheumatologists in screening for retinopathy in SLE patients, assessment of disease activity and treatment outcome.

Disclosure of Interest: None declared

DOI: 10.1136/annrheumdis-2018-eular.6783

\section{THU0371 CORRELATION BETWEEN IRREGULAR MENSTRUATION AND DISEASE ACTIVITY OF SYSTEMIC LUPUS ERYTHEMATOSUS :1 YEAR COHORT STUDY}

N. Oguro, N. Yajima, M. Saito. Rheumatology, Showa University School of Medicine, Tokyo, Japan

Background: Systemic lupus erythematosus (SLE) often occurs to young women of reproductive age. Not only the disease but the treatment itself could affect the capability of getting pregnant. In clinical scene, we often experience SLE patients having menstrual irregularity before their relapse. Reports about correlation between menstrual irregularity and disease activity of SLE was very limited.

Objectives: We started this study, that this study could be a suggestion for clinician to use the symptom as a warning sign for relapse.

Methods: The study design was prospective cohort.67 patients who fulfilled $\geq 4$ of the American College of Rheumatology (ACR) criteria for the classification of SLE whose age was 20 to 45 years old and treated as outpatient at Showa University Hospital from Feb 2016 to Jan 2017 were recruited. At the initial point, patients' disease were not active and were receiving maintenance therapy. Loss to follow up was eliminated. We set the main exposure as menstrual irregularity and the primary outcome as whether they would have relapse within 1 year from the registration. We defined the menstrual irregularity as deviation from the normal menstruation, which is 'flow occur every 25 to 35 days and last three to seven days'. We had questionnaire formed from each patient. Definition of relapse was the doctor's decision, to intensify the patients' treatment. We set the confounding as age, Japan Perceived Stress Scale (JPSS), current smoking, history of treatment with IVCY and logistic regression analysis was performed.

Results: The mean age was 35.7 years old. Irregular menstruation patients were $11(16.4 \%)$. The relapse group was $12(17.9 \%)$ and those of normal group was 59 $(89 \%)$. There was no significant difference in patients' background between both groups. Adjusted with the cited confounding variable there was no significant difference between the both groups (Odds ratio 2.25; 95\% Confidence Interval $(\mathrm{Cl})$ 0.24 to $20.7 ; p=0.47$ )
Abstract THU0371 - Table 1. Abstract THU0371 - Table 2. Logistic regression analysis. Adjusted with age, history of treatment with IVCY, current smoking, and JPSS.

\begin{tabular}{|c|c|c|c|c|}
\hline & & Normal menstruation & zular menstruati & \\
\hline & Total & group & eroup & p value \\
\hline Number & 67 & 56 & 11 & \\
\hline Age median [rQR] & $35.7[31-41]$ & $38[31 \div 41]$ & $37[28-41]$ & 0.35 \\
\hline 0 month SLEDAI median[IQR] & $3[2.6]$ & $2[0-6]$ & $4[2-6]$ & 0.24 \\
\hline JPSS median [TQR] & $15[11 \cdot 18.8]$ & $15[11 \cdot 18.5]$ & $13[9-29]$ & 0.51 \\
\hline Current smoking N(S) & $8(11.9)$ & $6(10.7)$ & $2(18.1)$ & 0.4 \\
\hline History of $\mathrm{IVCY} \mathrm{N}\left(\mathrm{s}_{\mathrm{s}}\right)$ & 10(149) & $8(14.2)$ & $2(18.1)$ & 0.74 \\
\hline 0 month PSL, dose median[IQQR] & $5.5[4 \cdot 10]$ & $5.7[4.3-9.6]$ & $5[3-11]$ & 0.73 \\
\hline 0 month Immune-Supprescing drug N( $(\varsigma)$ & 40(59) & $35(62.5)$ & $5(45.4)$ & 0.29 \\
\hline \multirow{2}{*}{\multicolumn{5}{|c|}{ 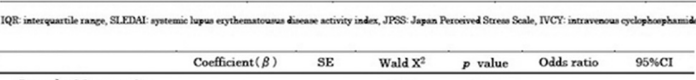 }} \\
\hline & & & & \\
\hline $\begin{array}{cc}\text { Irregular Menstruation } \\
\text { Group }\end{array} \quad-0.40$ & 0.56 & $\begin{array}{ll}0.51 & 0.47\end{array}$ & 2.25 & $0.24 \cdot 20.7$ \\
\hline
\end{tabular}

Conclusions: We investigate that whether menstrual irregularity could be the predictor for the disease relapse, but we could not prove the significant relation.

\section{REFERENCES}

[1] Shabanova SS. Ovarian function and disease activity in systemic lupus erythematosus, Clinical and Experimental Rheumatology 2008;26:436441.

[2] Silva CAA. Risk factors for amenorrhea in juvenile systemic lupus erythematousus(JSLE):A Brazilian multicenter cohort study. Lupus 2007;16:531536.

[3] LE Harward. The impact of cyclophosphamide on menstruation and pregnancy in woman with rheumatologic disease; Lupus 2013 22;81

[4] Wenhong Ma, Subclinical impairment of ovarian reserve in systemic lupus erythematosus patients with normal menstruation not using alkylating therapy. Journal of women's health,2013;22:4255

Disclosure of Interest: None declared

DOI: 10.1136/annrheumdis-2018-eular.5274

\section{THU0372 ULTRASONOGRAPHIC SCORING OF THE MAJOR SALIVARY GLANDS: IS THERE A RELATIONSHIP WITH DISEASE ACTIVITY AND FUNCTIONAL STATUS OF THE GLANDS ?}

${ }^{1}$ N. Inanc, Y. Yalcinkaya ${ }^{1}$, G. Mumcu ${ }^{2}$, Z. Erturk ${ }^{1}$, A.U. Unal ${ }^{1}$, P. Atagunduz ${ }^{1}$, H. Direskeneli'. ' Department of Internal Medicine, Division of Rheumatology, Marmara University, School of Medicine; ${ }^{2}$ University of Health Sciences, Istanbul, Turkey

Background: Ultrasonography (USG) of major salivary glands (SG-USG)is a non-invasive tool that has been used to evaluate salivary glands in primary and secondary Sjogren's syndrome (SjS).

Objectives: We aimed to investigate relation between the ultrasonographic scoring of major salivary glands and systemic disease activity or salivary secretion in patients with primary SjS.

Methods: Seventy-five SjS patients (F/M:73/2) with the mean age of $52 \pm 12$ and duration of follow-up period of $58 \pm 54$ months fulfilling ACR-EULAR classification criteria (2002) were included. Disease activity indexes (Sjögren's Syndrome Patients Reported Index (ESSPRI), Visual Analogue Scale (VAS), EULAR Sjögren's Syndrome Disease Activity Index (ESSDAI)] were recorded. Simultaneously, sialometric evaluation of the salivary glands was performed. Major salivary glands (bilateral parotis and submandibular glands) were scored according to two different scoring systems [Hocevar A.(0-48) and Milic VD. (0-12)] and elastography was recorded as well.

Results: Demographics, clinical characteristics, disease activity indexes and SG-USG scores were summarised in table 1 and table 2 . Forty-one (55\%) and 45 $(60 \%)$ patients had the cut-off values of $\geq 17$ (Hocevar) and $\geq 6$ (Milic USG). The patients with the scores of $>17$ (Hocevar) were found to have higher scores of ESSPRI-total $(16 \pm 6$ vs $13 \pm 7, p=0,045)$ and lower sialometry $(4,6 \pm 4,7$ vs 8,4 $\pm 4,6 \mathrm{ml}, \mathrm{p}=0,002)$. Scores of Hocevar and Milic-USG were negatively correlated with sialometry $(r=-, 430, p=0001$ and $r=-, 430, p=0,001)$. Hocevar, Milic and 
elastography-USG scores were shown to be higher in patients with sialometry of $\leq 1,5 \mathrm{ml}(n=7)(28 \pm 3$ vs $17 \pm 10, p=0,010,8 \pm 1$ vs $5 \pm 3, p=0006$ and $9 \pm 1$ vs $5 \pm 2$, $p=0,028)$ and anti-Ro positivity $(n=24)(24 \pm 10$ vs $13 \pm 87 \pm 3$ vs $4 \pm 2, p<0001$ and 7 \pm 2 vs $3 \pm 2, p=0,003$ ). The patients with severe parotid involvement (inhomogeneity/hypoechogenic areals $\geq 2$ ) had more frequent anti-Ro and anti-La positivity (80 vs $42 \%, p=0004$ and 48 vs $17 \%, p=0,011$ )

Abstract THU0372 - Table 1. Demographics and Clinical Characteristics of SjS patients.

\begin{tabular}{|l|c|}
\hline & $\mathrm{n}=75$ \\
\hline Sicca symptoms & $69(92 \%)$ \\
\hline Arthralgia/arthritis & $62(83 \%)$ \\
\hline Parotitis & $20(27 \%)$ \\
\hline Raynaud Phenomenon & $12(16 \%)$ \\
\hline Leucocytoclastic vasculitis & $5(7 \%)$ \\
\hline Peripheral neuropathy & $7(9 \%)$ \\
\hline Interstitial lung disease & $3(4 \%)$ \\
\hline Lymphadenopathy & $12(16 \%)$ \\
\hline Congenital heart block & $2(3 \%)$ \\
\hline ANA & $62(82 \%)$ \\
\hline Anti-Ro/La & $35(47 \%)$ \\
\hline ESR(mm/h) & $32 \pm 20$ \\
\hline CRP(mg/l) & $4,8 \pm 6,1$ \\
\hline
\end{tabular}

Abstract THU0372 - Table 2. Disease Activity Indexes and SG-USG Scores of SjS Patients

\begin{tabular}{|l|c|}
\hline & $n=75$ \\
\hline ESSPRI-total & $14,8 \pm 6,5$ \\
\hline -dryness & $5,4 \pm 2,5$ \\
\hline -fatigue & $4,8 \pm 2,8$ \\
\hline -pain & $4,6 \pm 3$ \\
\hline VAS ESSDAl-total & $50 \pm 22$ \\
\hline Hocevar-USG Score & $2,1 \pm 3,5$ \\
\hline Milic-USG Score & $19 \pm 5$ \\
\hline Sialometry (ml) (n=45) & $6 \pm 3$ \\
\hline Elastography ( $n=32)$ & $6,4 \pm 5$ \\
\hline
\end{tabular}

Conclusions: Hocevar scoring system of major salivary glands was found to be related to patient reported activity in SjS. USG scores were associated with reduced saliva secretion and anti-Ro positivity. Severe parotid involvement was shown to be related to anti-RO and La positivity. Evaluation of SG-USG including different scoring systems and elastography might reflect function of the salivary glands.

Disclosure of Interest: None declared

DOI: 10.1136/annrheumdis-2018-eular.7099

\section{THU0373 THE MARKERS USEFUL IN PREDICTING LUPUS NEPHRITISIN CLINICAL PRACTICE}

${ }^{1}$ P. Dabrowski, K. Marczak ${ }^{1}$, A. Gala-Bladzińska ${ }^{2,3}$, K. Gargasz ${ }^{4}$, M. Dabrowska ${ }^{5}$. ${ }^{1}$ Department of Rheumatology, Clinical Provincial Hospital No. 2, Lwowska 60 Street, 35-301 Rzeszów, Poland; ${ }^{2}$ Faculty of Medicine, University of Rzeszów, Kopisto 2a Avenue, 35-359 Rzeszów, Poland; ${ }^{3}$ Dialysis Center, Clinical Provincial Hospital No. 2, Lwowska 60 Street, 35-301 Rzeszów, Poland; ${ }^{4}$ Data Analysis Laboratory, Centre for Innovative Research in Medical and Natural Sciences, Faculty of Medicine, University of Rzeszow, Kopisto 2a Avenue, 35-359 Rzeszów, Poland; ${ }^{5}$ II Department of Pediatrics, Endocrinology and Diabetology, Clinical Provincial Hospital No. 2, Lwowska 60 Street, 35-301, Rzeszów, Poland

Background: Lupus nephritis (LN) is one of the most severe clinical manifestations of systemic lupus erythematosus (SLE). LN can be found in approximately $50 \%$ of SLE patients. The renal biopsy remains the gold diagnostic standard. However non-invasive and clinically practical laboratory markers of kidney damage in this disease are sought.

Objectives: The aim of the study was to assess the utility of biomarkers like inflammable indicators, complement system components and albuminuria in a single urine sample for prediction of kidney involvement and disease activity in patients with SLE.

Methods: A prospective study included 33 patients (81.8\% women) with SLE criteria according to SLICC (Systemic Lupus International Collaborating Clinics) in age of $39.0 \pm 14.3$ years. Disease-duration ranged $6-60$ months. We performed full physician examination and we excluded patients with active infection. The disease activity based on the SELENA-SLEDAI scale was assessed and divided into groups: low activity $(L)<6$, moderate $(M) 7-12$, high $(H)>12$ points.

In the blood samples complement components (C3, C4) (g/L), C-reactive protein CRP $(\mathrm{mg} / \mathrm{L})$, interleukin $6(\mathrm{IL}-6)(\mathrm{pg} / \mathrm{mL})$, serum creatinine concentration $(\mathrm{sCr})$ $(\mathrm{mg} / \mathrm{g})$, ESR $(\mathrm{mm} / \mathrm{h})$, glomerulal filtration rate (eGFR) according to CKD-EPI $(\mathrm{ml} /$ $\mathrm{min} / 1.73 \mathrm{~m}^{2}$ ) were determined. The concentration of albumin (uAlb) (mg/dl) and creatinine $\mathrm{sCr}(\mathrm{g} / \mathrm{dL})$ from the morning urine sample was measured and the albu $\mathrm{min} /$ creatinine index (UACR) $(\mathrm{mg} / \mathrm{g}$ ) was calculated. Based on the obtained results, patients were divided into stages of chronic kidney disease (CKD) according to .KDIGO 2012. A daily proteinuria (DP) (g/24 hour) was performed. In the assessment of statistical significance, Kruskal-Wallis or Mann-Whitney U-tests were used.

Results: In our study the SLE activity was as follows (\%): L-24 (72.7), M-6 (18.2), $\mathrm{H}-3$ (9.1). The average values (range) of biomarkers of renal function were: $\mathrm{Cr}=0,81 \pm 0,27(0.55-1.65), \mathrm{eGFR}=99.6 \pm 24.4(46-131), \mathrm{uAlb}=13.6 \pm 34.4(0.04$ 161.0), uACR=121.3 \pm 356.3 (4.8-1905.3), $D P=0.32 \pm 0.92(0.015-5.3)$, and other biomarkers: $\mathrm{OB}=26.1 \pm 25.9$ (4.0-99.0); $\mathrm{CRP}=10.3 \pm 28.1 \quad(0.2-148.7) ; \mathrm{IL}-6=7.8$ \pm 14.7 (0-78.3); C3=1.08 $\pm 0.36(0.33-2.25)$; C4=0.16 $\pm 0.09(0.02-0.43)$. The study group met the CKD criteria: G1 $n=21(63.6 \%), G 2 n=9(27.3 \%), G 3 n=3(9.1 \%)$ $A 1 n=26(78.8 \%), A 2 n=3(9.1 \%), A 3 n=4(12.1 \%)$.

We showed a negative relationship between the eGFR and: CRP ( $R=-0.49$ $\mathrm{p}=0.005)$, IL-6 $(\mathrm{R}=-0.48, \mathrm{p}=0.005)$ and $\mathrm{C} 4(\mathrm{R}=-0.43, \mathrm{p}=0.01)$. There was also a significant dependence of the SLEDAI SLE activity with: $u A l b(L, H)(p=0.04)$, DP $(\mathrm{M}, \mathrm{H})(\mathrm{p}=0.03), \mathrm{uACR}$ in the whole study group $(\mathrm{p}=0.04)$ and between $\mathrm{ACCR}$ and DP $(p=0.0003)$.

Conclusions: Our studies showed that the risk of kidney damage in SLE may depend on the concentration of CRP, IL-6, C4. In addition albuminuria (UAlb, UACR) correlates with the value of DP and SLE activity, what indicates the dominant glomerular lesion in the etiopathogenesis of proteinuria in $\mathrm{LN}$.

Disclosure of Interest: None declared

DOI: 10.1136/annrheumdis-2018-eular.5867

\section{THU0374 FACTORS ASSOCIATED WITH HIGH-DOSE CORTICOSTEROID USE IN SLE PATIENTS POST INITIATION OF SLE THERAPY}

K. Schroeder ${ }^{1}$, J. Paik ${ }^{1},{ }^{1}$ R.W. Hoffman, S. Gelwicks ${ }^{2} .{ }^{1}$ Eli Lilly and Company; ${ }^{2}$ Former Eli Lilly and Company Employee, Indianapolis, USA

Background: Systemic lupus erythematosus (SLE) therapies include non-steroidal anti-inflammatory drugs, antimalarials, systemic immunosuppressants, and biologics with corticosteroids as necessary. The majority of these current therapies are only partially effective in disease control. Despite treatment, patients may experience flares of disease activity, which can lead to progressive end-organ damage. Severe flares may require intensive immunosuppression, including with high-dose corticosteroids, with risk including end-organ damage.

Objectives: To understand the unmet need in SLE by quantifying use of highdose ( $\geq 40 \mathrm{mg} /$ day) corticosteroids and determining factors associated with its use.

Methods: This study utilised the Truven Marketscan commercial claims database. Patients were indexed on first use of antimalarial, oral immunosuppressant or biologic during 2012-2013 (first use determined based on no claims for the 3 drug classes during the 1 year pre-index). Included patients had 2 recorded SLE diagnoses, were 18-50 years of age and had continuous medical and prescription enrollment from baseline through the 2 year follow-up. Patients with other prespecified autoimmune disorders or cancers during the study period (baseline through follow-up) were excluded. During follow-up, fill of at least 1 high-dose corticosteroid prescription was assessed and associative logistic regression modelling performed.

Results: 1401 patients (93\% female; mean age 38.4 years) met the study criteria; $79 \%$ were indexed on an antimalarial, $15 \%$ on an oral immunosuppressive, $1 \%$ on a biologic and $5 \%$ on a combination of at least 2 of the aforementioned classes. $16 \%$ patients received a diagnosis code for nephritis or chronic kidney disease (CKD), $3 \%$ for myocarditis or pericarditis, and $13 \%$ for thrombocytopenia or leukopenia. During baseline, $56 \%$ of patients had at least 1 visit to a rheumatologist and $13 \%$ filled at least 1 high-dose corticosteroid prescription. During follow-up, $22 \%$ of patients had at least 1 high-dose corticosteroid prescription. Factors significantly associated $(p<0.05)$ with high-dose corticosteroids during follow-up included: baseline rheumatologist visit ( $\mathrm{OR}=0.62 ; 95 \% \mathrm{Cl}=0.47-0.82)$, number of SLE medication classes received during follow-up $(\mathrm{OR}=1.85,95 \% \mathrm{Cl}=1.36$ $2.51)$, receipt of high dose corticosteroid during baseline $(\mathrm{OR}=5.21,95 \%$ $\mathrm{Cl}=3.60-7.53)$, nephritis or $\mathrm{CKD}(\mathrm{OR}=1.85,95 \% \mathrm{Cl}=1.29-2.64)$, myocarditis/pericarditis (OR=3.38, 95\% Cl=1.75-6.55), and thrombocytopenia/leukopenia $(\mathrm{OR}=1.70,95 \% \mathrm{Cl}=1.17-2.48)$.

Conclusions: A number of baseline factors were associated with high-dose corticosteroid treatment during the follow-up period; one notable factor is the high percentage of patients using high-dose corticosteroids ( $\geq 40 \mathrm{mg} /$ day). This indicates 